\title{
Mass spectrometry based analysis of erythrocyte membrane associated proteins in chronic myeloid leukemia patients in Sri Lanka
}

\author{
D. U. Kottahachchi ${ }^{1, *}$, T. R. Ariyaratne ${ }^{1}$, G. A. U. Jayasekera ${ }^{2}$ \\ ${ }^{1}$ Department of Physics, Faculty of Science, University of Colombo, Sri Lanka \\ ${ }^{2}$ Department of Plant Sciences, Faculty of Science, University of Colombo, Sri Lanka \\ *E-mail address: dkottahachchi@yahoo.com
}

\begin{abstract}
The research reported in this paper was conducted to analyze erythrocyte membrane associated proteins (ERMBPs) of some of chronic myeloid leukemia (CML) patients and selected individuals of Sri Lanka employing one dimensional sodium dodecyl sulphate poly acrylamide gel electrophoresis (1D-SDS-PAGE) combined with matrix-assisted laser desorption ionization time of flight mass spectrometry (MALDI-TOF-MS). Erythrocyte membranes from blood were isolated by osmotic lysis, centrifugation and washings. ERMBPs were separated on 1D-SDS-PAGE, visualized by silver staining and the separated protein bands dissected out from the gel and were subjected to digestion by proteolytic enzyme, trypsin, and the resulting peptide mixture was analyzed by MALDI-TOF-MS. Resulting experimental peptide mass values were analyzed by peptide mass fingerprint (PMF) technique. From this analysis 10 ERMBPs including $\alpha$ and $\beta$ spectrin, ankyrin, band 3, band 4.1, band 4.2 , band 7, dematin, actin, $55 \mathrm{KDa}$ erythrocyte membrane protein were identified accurately with their primary structure information. The study was able to provide some evidence for Cathepsin associated cleavage of Band 3 anion transport protein in CML patients reported previously. In addition we were able to detect changes in gel bands between healthy controls and CML patients around the area of $20 \mathrm{kDa}$ in the $1 \mathrm{D}$-SDS-PAGE. It was identified as nuclear protein $\mathrm{Dbf} 4$ related factor 1 isoform 2. Although erythrocytes are devoid of nuclei, such unexpected nuclear proteins have been identified in previous research. We were successful in identifying several human ERMBPs with available resources. As the identified proteins were known to be related to pathology of some of the hematological diseases, this methodology could be extended to detect the protein changes in erythrocyte membrane protein associated diseases. Therefore, this initial research would at some point lead to discovery of biomarkers to these hematological diseases in Sri Lanka.
\end{abstract}

Keywords: Erythrocyte membrane associated proteins; 1-D-SDS-PAGE; MALDI-TOF-MS; PMF; Hematological diseases

\section{INTRODUCTION}

Cells as a cause or consequence of pathological conditions increase or decrease its protein expressions. Study of the protein complement of a genome / or global protein expression of human cells is a rapidly growing research area that has tremendous potential to unravel basic biological questions regarding mechanisms of diseases and potential usefulness 
as a diagnostic tool. Proteins are responsible for membrane functions such as transport, enzymes, signaling, cell-cell recognition, intercellular joining, and attachment. Membrane proteins are among the most important proteins biologically because they allow the cells to communicate with their environments and play key roles in controlling the cellular functions and processes of life ${ }^{(1)}$.

Human erythrocyte membrane associated proteins (ERMBPs) play a pivotal role during its critical functions. The membrane proteins exhibit diverse functional heterogeneity, serving as transport proteins, as adhesion proteins involved in interactions of red cells with other blood cells and endothelial cells, as signaling receptors, and other still undefined activities ${ }^{(2)}$.

Chronic myeloid leukemia (CML) is a clonal disorder of a Pluripotent stem cell (PSC) and is classified as one of the myeloproliferative disorders. It is usually a disease of the elderly, although it can occur at any age. There are excessive accumulations of neoplastic granulocytes and their precursors, principally in the bone marrow, spleen, liver and blood. The disease is insidious some are even diagnosed incidentally through routine blood studies. The prognosis is commonly fatal with an average survival time of three to five years ${ }^{(3)}$.

Erythrocyte membrane associated proteins have subjected investigation of several hematological disorders in humans ${ }^{(4)}$. It has been reported that several erythrocyte membrane associated proteins demonstrate quantitative as well as qualitative changes as a result of leukemia. For example it has been reported that erythrocyte membrane associated protein Kinase $\mathrm{C}$, and band 3 protein show alterations in chronic myelogenous leukemia (CML) patients when compared to normal erythrocytes ${ }^{(5)}$. In addition it is reported that the CML causes abnormalities in erythrocyte membrane associated spectrin protein as well ${ }^{(6)}$. Recently, Govekar et al. (2012) reported that the role of neutrophilic cathepsin G, detected as an immunogen on erythrocyte membrane, in band 3 cleavage. Cathepsin $G$ from serum adsorbs to the erythrocyte membrane to mediate cleavage of band 3 and therefore contribute to the eryptotic phenotype in $\mathrm{CML}^{(7)}$.

In recent years, the technology and methods widely available for mass spectrometry (MS)-based proteomics have increased in power and potential, allowing the study of proteinlevel processes occurring in biological systems. Matrix Assisted Laser Desorption Ionization Time Of Flight Mass Spectrometry (MALDI-TOF MS) is a well-established MS method that permits analysis of virtually all proteins of a complex mixture resolved by gel electrophoresis $^{(8)}$. MALDI TOF technique employs the co crystallization of matrix (low molecular weight organic crystalline compound) with the analyte (protein) on a metal target plate. Irradiation of these crystals by pulse laser ion source initiates desorption and ionization, where predominantly singly protonated intact molecular ions $\left[\left(\mathrm{M}^{+} \mathrm{H}\right)^{+}\right]$are produced. During this process bunches of ionized, protonated proteins are accelerated through electric field towards the detector. The time taken by the bunch of ions to reach the detector or the Time Of Flight is inversely proportional to charge and directly proportional to the mass ${ }^{(9)}$. Several improvements to matrix-assisted laser desorption ionization time of flight mass spectrometry (MALDI-TOF-MS) instrumentation coupled with improved protein separation techniques such as one dimensional sodium dodecyl sulphate poly acrylamide gel electrophoresis (1DSDS-PAGE) and/or two dimensional poly acrylamide gel electrophoresis (2D-PAGE) have been made over the past few years ${ }^{(9)}$. These techniques coupled with peptide mass fingerprinting (PMF) facilitates identification of proteins of interest and it is currently accepted as one of the major techniques for analysis of proteins in a mixture ${ }^{(10)}$.

Recent technological advances that include proteomics and microarray methods have allowed scientists to study global approaches for protein identification and gene expression analysis in cells. The absence of a nucleus in the mature erythrocyte has made mRNA based 
micro array based approaches not applicable for erythrocyte. However mass spectrometry associated proteomics technologies have permitted fast and reliable detection and identification of erythrocyte proteins somewhat offsetting the drawbacks caused due to the lack of microarray technology ${ }^{(11)}$.

In this paper we present the data from research conducted to analyze erythrocyte membrane protein profiles of some of chronic myeloid leukemia (CML) patients and selected healthy individuals of Sri Lanka. ERMBPs were chosen for this study as they are readily available in large amounts and also had been well characterized biochemically in previous studies. To our knowledge this work represent, a first time that PMF combined with mass spectrometric technology has been employed for ERMBPs in leukemia patients in Sri Lanka.

\section{MATERIALS AND METHODS}

Blood samples from 7 chronic myeloid leukemia (CML) patients were obtained from Government Cancer Hospital, Maharagama, Sri Lanka after obtaining their consent. All the patients were above 40 years of age and samples were collected at the diagnosis and have not been treated. Their white blood cell counts (WBC) were ranging from $50-80 \cdot 10^{3} / \mathrm{L}$ and Hemoglobin $(\mathrm{Hb})$ values were ranging from 10.0-11.0 g/dl. Hematocrit (HCT) values were ranging from $31-33 \%$. Platelet counts were approximately $400 \cdot 10^{9} / \mathrm{L}$. Blood of 6 healthy persons were obtained from volunteers from the staff members at Cancer hospital. All of them had normal WBC, Hb, HCT and platelet counts. The Ethical clearance was approved by the Ethical Review Committee of Faculty of Medicine, University of Colombo (Reference No: EC/04/079). Blood was mixed with $4 \%$ w/v EDTA, pH 7.4 as anticoagulant at a ratio of 1:20. All the chemicals were purchased from Sigma (St. Louis, MO, USA).

The erythrocyte membranes were isolated by washing, osmotic lysis and centrifugation of erythrocytes as described in Low et al, 2002 ${ }^{(12)}$. Finally the pellet was dissolved in dissolving buffer cocktail consisting of $1 \%$ SDS, 5-10 \% Sucrose, $10 \mathrm{mM}$ Tris- $\mathrm{HCl}$ (pH 8.8), $1 \mathrm{mM}$ EDTA (pH 8.0) and 40mM DTT. Total protein amounts were estimated by Lowry method (DC Protein assay, Bio-Rad laboratories, Richmond, USA) and Bovine serum albumin (BSA) was used as a standard.1D-SDS-PAGE was performed according to Laemmli's Tris-glycine system ${ }^{(12)} .60 \mu \mathrm{g}$ of proteins were loaded into each well in the gel and electrophoresis was performed until the dye reaches to the bottom of the plate. Then the gel was soaked in the fixative containing ethanol: acetic acid: water (4: 1: 5) for 30-45 minutes and followed by de-ionized water at room temperature for overnight. 1D-SDSPAGE gels stained manually with silver. Minute amount $(15 \mathrm{mg} / \mathrm{L})$ of sodium thiosulphate pentahydrate and $50 \mathrm{~g} / \mathrm{L}$ Tris base were included in the developing solution and stop solution in order to improve the quality of staining. Distinct gel bands as well as bands with differential intensities between patients and controls were excised from the gel, were treated with $100 \mathrm{mM}$ sodium thiosulfate: $30 \mathrm{mM}$ potassium ferricyanide (1:1) for a few minutes and $0.05 \mathrm{M}$ ammonium bicarbonate and $100 \%$ acetonitrile washes to improve the MALDI-TOF spectra by removing silver. Finally, they were dried on a speedvac for 20-30 min. In-gel reduction, alkylation and trypsin digestion of proteins were performed ${ }^{(12)}$. A solution of 12.5 $\mathrm{ng} / \mu \mathrm{L}$ bovine trypsin in $0.05 \mathrm{M}$ ammonium bicarbonate was added to each tube and kept on ice for 30 min., sufficient amount of buffer solution was added to cover the gel pieces, kept at $37{ }^{\circ} \mathrm{C}$ overnight for further digestion. Next, the extraction of peptides was carried out to collect more peptides ${ }^{(12)}$. Finally, the solution was completely dried in a speedvac and re dissolved with $1 \%$ formic acid just before MS analysis. In MALDI-MS, the protein and 
peptide samples were embedded in a matrix that absorbs the laser light. The matrix solution consisted of saturated $\alpha$ cyano - 4- hydroxycinnamic acid with $50 \%$ acetonitrile $+0.1 \%$ TFA. MALDI-MS sample preparation was performed according to the dried droplet method. In this method $0.3 \mu \mathrm{L}$ of the eluting matrix was put to the target metal plate. Subsequently 0.3 $\mu \mathrm{L}$ of the sample (extracted solution) was put to the same spot and allowed to dry ${ }^{(9)}$.

Next, MS analysis was performed using mass spectrometer Bruker Daltonics (Germany) at Ludwig Institute for Cancer Research, Bio Medical Centre (BMC), Uppsala University, Sweden. Target metal plate was introduced into the mass spectrometer and time of flight was measured using the following specifications. The MS automatic data acquisition was performed in delayed extraction, reflector mode using an acceleration voltage of $20 \mathrm{KV}$; Grid voltage $70 \%$ and $100 \mathrm{~ns}$ delay. Desorption and ionization of samples occurred with a nitrogen laser $(\lambda=337 \mathrm{~nm})$ and laser intensity was set at 2000 with 100 shots per spectrum. The mass range was set between $700-3500 \mathrm{Da}$. The bovine trypsin auto digested peptides (805.416 Da, 1020.503 Da, 1111.560 Da, 1433.720 Da, 2163.056 Da, 2273.159 Da $\left[\mathrm{M}^{+} \mathrm{H}^{+}\right]$ ions) were set as internal calibration standards. Finally, proteins were identified by matching our experimental mass values with theoretical mass values in the database. The identification of the relevant protein was made with a statistical scoring procedure using the following web site http://www.expasy.ch/tools/peptide-mass.html, which is freely available in Internet. It was used to compare the molecular weight, proteolytic digest fragment, and sequence information obtained for the erythrocyte membrane associated proteins to known proteins in the Swiss-Prot and NCBI databases ${ }^{(10)}$.

\section{RESULTS}

\section{1. Analysis of erythrocyte membrane associated proteins of healthy controls and CML patients}

1D-SDS-PAGE protein profiles were studied in seven CML patients and six healthy controls. Figure I shows the silver stained gel which contains representative samples of resolved erythrocyte membrane associated proteins of one healthy control and two CML patients. Selected protein bands from healthy controls and CML patients were successfully identified with mass spectrometric techniques and database search. $R_{1}$ to $R_{10}$ from one healthy control in Figure I were analyzed by MALDI-TOF and identification was made using database programs by employing PMF. Identified proteins were annotated to their locations using Scaffold software (version 3.3.2, Proteome software Inc.). The identification and the annotation revealed that these proteins were located either erythrocyte membrane or membrane associated.

The MALDI-TOF spectrum of $\mathrm{R}_{1}$ protein (in Fig. I) is shown in Figure IIa. The $\mathrm{R}_{1}$ protein was identified as Spectrin, alpha chain, erythrocyte (Erythroid alpha- spectrin) gi/1174412/sp/P02549/SPCA_HUMAN with $15 \%$ sequence coverage. The molecular mass is $281.04 \mathrm{kDa}$ and the corresponding masses $\left[\mathrm{M}^{+} \mathrm{H}\right]$ for mono isotopic masses [M] of 1340.634 Da, 1545.701 Da in matched peptides were observed in the MALDI-TOF spectrum (Figure IIa). In addition, the sequence information of the two matched peptides were obtained and shown in Table 3. The MALDI-TOF spectrum of $\mathrm{R}_{2}$ protein (in Fig. I) is shown in figure IIb. $\mathrm{R}_{2}$ protein was identified as Spectrin, beta, erythrocytic [Homo sapiens] gi/174798/sp/P11277/ SPCB_HUMAN with $8 \%$ sequence coverage. The molecular mass is $247.16 \mathrm{kDa}$ and the corresponding masses $\left[\mathrm{M}^{+} \mathrm{H}\right]$ for mono isotopic masses $[\mathrm{M}]$ of 848.416 $\mathrm{Da}, 1113.520 \mathrm{Da}$ in matched peptides were observed in the MALDI-TOF spectrum (Figure 
IIb). In addition, the sequence information of the two matched peptides were obtained and shown in Table 3. The following common erythrocyte membrane proteins were resolved by 1D-SDS-PAGE as shown in Figure $I$ and were identified using the above described procedure: Spectrin beta chain, erythrocyte $\left(\mathrm{R}_{2}\right.$ in Fig. I), Ankyrin-1 $\left(\mathrm{R}_{3}\right.$ in Fig. I), Band 3 anion transport protein $\left(\mathrm{R}_{4}\right.$ in Fig. I), Erythrocyte membrane protein band 4.1 ( $\mathrm{R}_{5}$ in Fig. I), Erythrocyte membrane protein band 4.2 ( $\mathrm{R}_{6}$ in Fig. I), $55 \mathrm{kDa}$ erythrocyte membrane protein $\left(\mathrm{R}_{7}\right.$ in Fig. $\left.\mathrm{I}\right)$, Dematin $\left(\mathrm{R}_{8}\right.$ in Fig. $\left.\mathrm{I}\right)$ and Erythrocyte band 7 integral membrane protein $\left(\mathrm{R}_{10}\right.$ in Fig. I). Table I lists the database search results for the identities of each of these protein bands. It shows gel band number for each band, the protein corresponding protein, the respective accession numbers, sequence coverage and the molecular weights of the identified protein.

The results in Table 1 illustrate clearly, the extent in which the technology used in this study was successful in identifying ERMBPs belonging to a broad molecular weight range (from $31-281 \mathrm{kDa}$ ). This reiterates the dependability and the reliability of MALDI-TOF \& PMF in identification of resolved proteins from biological samples such as blood. Furthermore, their amino acid sequences were obtained and the corresponding masses $\left[\mathrm{M}^{+} \mathrm{H}\right]$ for mono isotopic masses [M] could be observed in the mass spectrums (data not shown) as same as explained in the $\alpha$ spectrin and $\beta$ spectrin. We failed to detect rest of the protein bands. There might be several reasons leading to this result, such as lower abundance, lower reliability of the MS results, and some characteristics of these proteins.

Next, the protein bands present in CML patients (in Fig. I) were compared with healthy controls. Figure I show some of clear intensity differences of protein bands between CML patients and healthy controls. Those differences were analyzed by MALDI-TOF MS \& PMF and were identified using database search. $\mathrm{R}_{\mathrm{X}}$ from healthy controls was identified as Dbf4 related factor 1 isoform 2 with a molecular weight of $18.60 \mathrm{kDa}$ (Table 1). It was not detectable in 2 CML patients (Figure 1). This observation was evident in all the healthy controls and CML patients used in the study. $\mathrm{R}_{4}$ which was identified as Band 3 anion transport protein in healthy controls and was not detectable in the parallel gel area of two CML patients as shown in Figure I. A distinct band appears around $55 \mathrm{kDa}$ area of CML patients (highlighted in arrows), most likely to be a fragment of $\mathrm{R}_{4}$ as described in Goveka et al. 2012. This observation was evident in all the healthy controls and CML patients used in the study. However, we failed to identify it.

\section{DISCUSSION}

Valuable insights into cellular functions and the corresponding gene functions can be gained by studying gene expression at the mRNA or protein level. Analysis of proteins has advantages since proteins are the final functional molecules that serve as the core of the functional machinery in the cell. The entire complement of a cell or proteome reflects the various functions performed within it. Analysis of proteins in a cell and tissues helps to gain insight into the molecular mechanism in various cellular processes and unraveling basic biological questions. This knowledge could lead to better understanding of cellular mechanisms that may result in clinically important diseases. The identification of gel separated unknown proteins is of paramount importance in many areas of biological research. The development of mass spectrometry and the evolution of algorithms that can correlate the data obtained from MS techniques like MALDI - TOF analysis with protein and genomic 
databases enable one to use PMF information to establish the identity of gel separated unknown proteins.

Blood samples of CML patients and healthy individuals (controls) were kept at $+4{ }^{\circ} \mathrm{C}$ for 3 days in order to mature reticulocytes to erythrocytes. Since reticulocytes having nuclear remnants and proteins in the nuclear remnants may interfere with the ERMBPs. The crucial step of the study was to prepare erythrocyte membranes from whole blood as the membrane fraction was less compared to the whole cell. This was efficiently carried out by first,

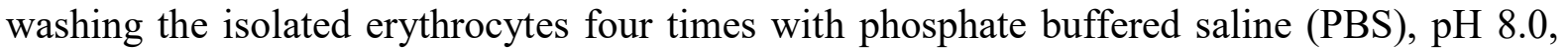
second, isolating erythrocyte membrane fractions by osmotic lysis of washed erythrocytes, followed by several high speed centrifugations, and washing steps ${ }^{(12)}$.

40-50 gel bands were detected using 1D-SDS-PAGE followed by silver staining (Figure I). In this Study we were able to detect both highly abundant and less abundant gel bands representing wide range of molecular weight area of 10-300 KDa. The first comprehensive study of the erythrocyte membrane proteome was reported by Low et al. $(2002)^{(12)}$ employing both 1 D-SDS-PAGE and 2 D-PAGE coupled with MALDI-TOF-MS in which 84 distinct RBC membrane proteins were successfully identified. However they were failed to identify most of the erythrocyte transmembrane hydrophobic proteins such as glycophorins. We confined the study mainly to identify highly abundant protein bands and few low abundance protein bands due to limited availability of resources at our disposal. We were successful not only in identifying 10 ERMBPs from healthy persons but also to detect the protein changes between healthy persons and CML patients (Table 1). However we failed to identify low abundance proteins (data not shown) due to limitation of the mass spectrometer that was used in this study.

The ERMBPs identified in this study $\left(\mathrm{R}_{1}-\mathrm{R}_{10}\right)$ are reported to contribute towards the maintenance of integrity the cell structure and shape. For example the two isoforms of spectrin, $\alpha$ and $\beta$, the major membrane associated proteins in human red blood cells play an important role in maintaining its round biconcave shape. They are present in tetramers and organized into a meshwork that is fixed to the membrane by the protein ankyrin. Ankyrin itself is connected to a transmembrane protein called 'band 3' or anion exchanger protein. The purpose of band 4.2 may be to stabilize the link between ankyrin and the anion exchanger. Spectrin is also linked to a transmembrane protein called glycophorin $\mathrm{C}$ by the protein known as 'band 4.1.' Thus the meshwork is anchored to the membrane at multiple sites. Band 4.1 stabilizes the association of spectrin with actin. Dematin also had known as band 4.9 protein, crosslink some of the actin microfilaments to make bundles of $\mathrm{F}$ (filamentous) actin ${ }^{(3)}$.

Investigation of molecular basis of some genetic disease of erythrocytes have revealed that several of these diseases are linked to abnormalities in ERMBPs which could range from a defective protein, to total absence or to an alteration in abundance due to reduced expression of a protein ${ }^{(4)}$. In several of these hematological diseases, abnormalities in ERMBPs leads to changes in shape of RBCs which get destroyed in extravascular sites such as spleen and liver before their normal life span. It has been reported that lower levels in abundance of spectrin (alpha, beta) and/or ankyrin-1, band 3 anion transport protein, erythrocyte membrane protein 4.2 were observed in Hereditary Spherocytosis (HS) ${ }^{(13)}$. 


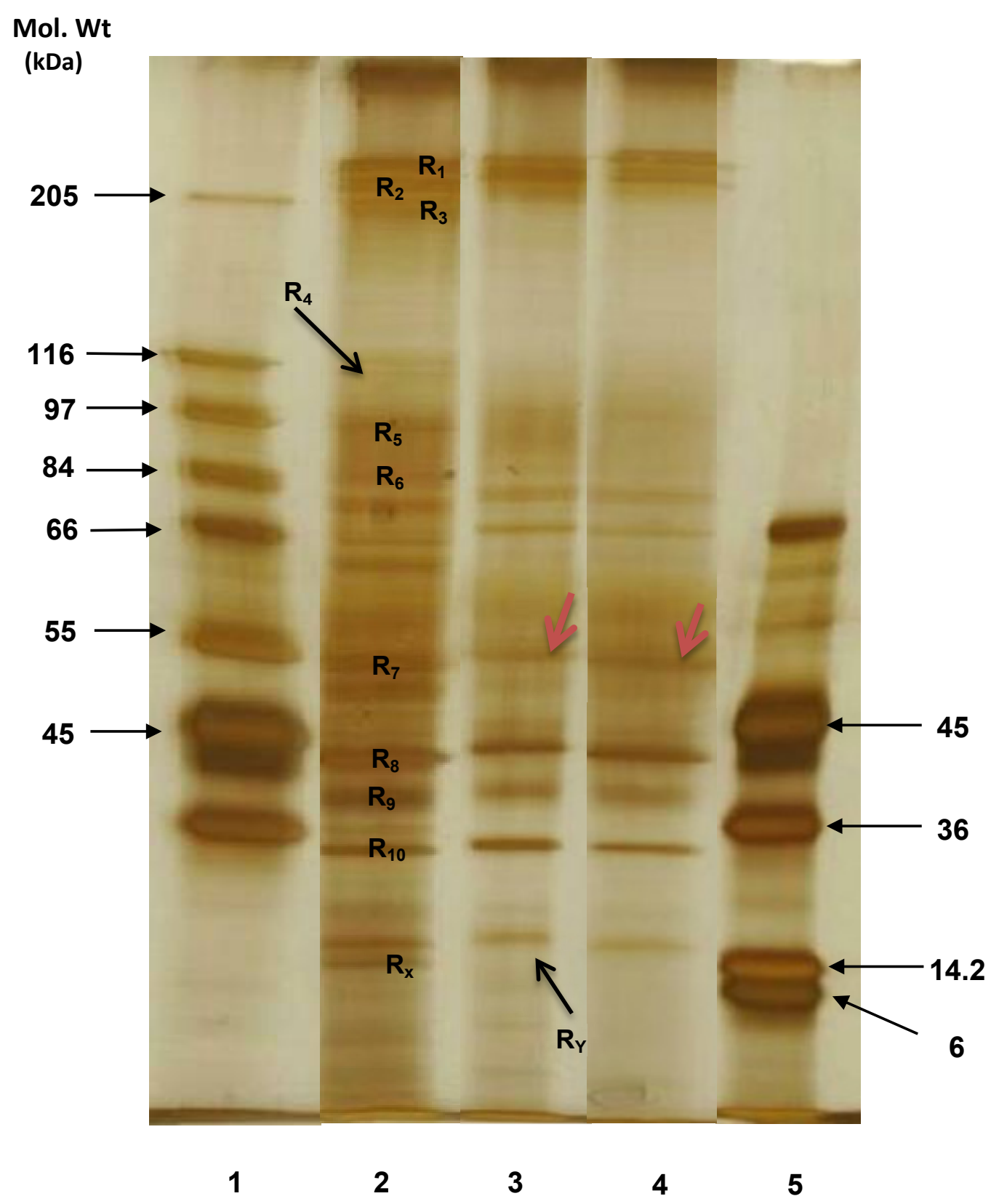

Figure I. 1-D SDS-PAGE (12\%) silver stained gel containing erythrocyte membrane associated proteins of normal healthy controls and chronic myeloid leukemia (CML) patients. $60 \mu \mathrm{g}$ of proteins were loaded in to each gel and subjected to electrophoresis. Lane 1: High Molecular weight marker (36-205 kDa); Lane 2: Normal RBC membranes; Lane 3: RBC membranes CML patient 1; Lane 4: RBC membranes CML patient 2; Lane 5: Low Molecular weight marker $(6-66 \mathrm{kDa})$. Gel bands of $\mathrm{R}_{1}-\mathrm{R}_{10}$ were analyzed by MALDI-TOF \& PMF and identified as erythrocyte membrane associated proteins. $R_{x}$ was detected only in the healthy control gel lane and it was identified as Dbf4-related factor 1 isoform 2; activator of S-phase kinase-like protein by MALDI TOF and PMF. $\mathrm{R}_{\mathrm{Y}}$ (in CML patient) was analyzed in order to confirm the absence of proteins in the area parallel to Rx. Highlighted bands (K) appears around $55 \mathrm{kDa}$ area of CML patients most likely to be a fragment of $\mathrm{R}_{4}$ and they were failed to identify. 

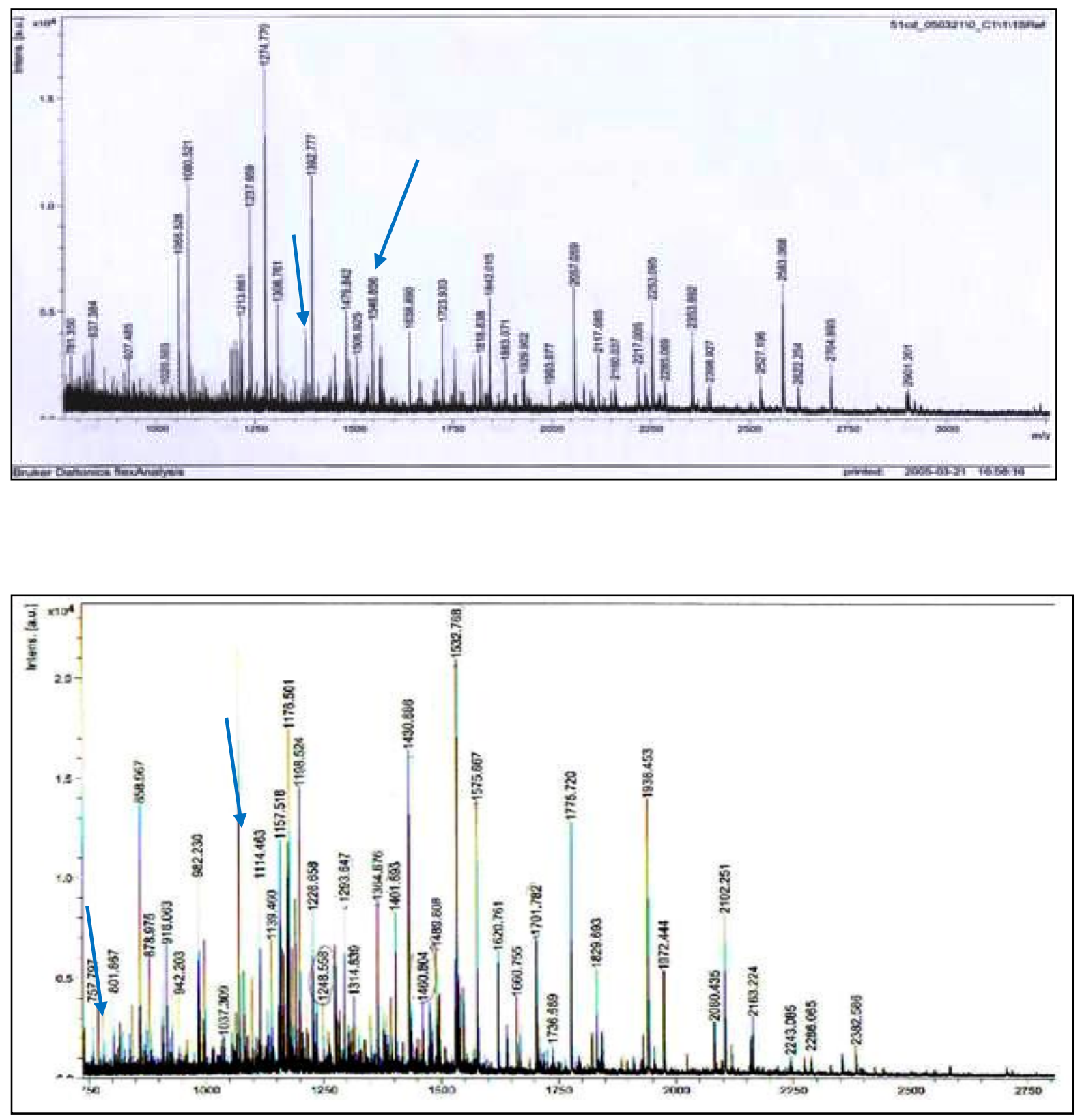

Figure II. MALDI-TOF MS spectrums of in-gel trypsin digested silver stained protein bands in Figure I. (a) MALDI-TOF mass spectrums of $\mathrm{R}_{1}$ protein band in Figure 5. There were 62 mass peptides used in the search and 27 were matched with the theoretical peptide masses in the database. The corresponding masses $\left[\mathrm{M}^{+} \mathrm{H}\right]$ for mono isotopic masses $[\mathrm{M}]$ of $1340.634 \mathrm{Da}, 1545.701 \mathrm{Da}$ in matched peptides were observed in the MALDI-TOF spectrum (highlighted in arrow). (b) MALDITOF mass spectrums of $\mathrm{R}_{2}$ protein band in Figure I. There were 136 mass peptides used in the search and 18 were matched with the theoretical peptide masses in the database. The corresponding masses $\left[\mathrm{M}^{+} \mathrm{H}\right]$ for mono isotopic masses $[\mathrm{M}]$ of $848.416 \mathrm{Da}, 1113.520 \mathrm{Da}$ in matched peptides were observed in the MALDI-TOF spectrum (highlighted in arrow). Common trypsin peptides masses $\left[\mathrm{M}^{+} \mathrm{H}\right]$ 1020.503 $\mathrm{Da}$ and 2163.224 Da were used for the internal calibration.

Reduction in abundance levels of spectrin (alpha, beta) and/or ankyrin-1, glycophorin $\mathrm{C}$, erythrocyte membrane protein 4.1 were reported to be associated with Hereditary 
Elliptocytosis (HE). Reduced levels of spectrin (alpha, beta), ankyrin-1, glycophorin C, erythrocyte membrane protein 4.1 were reported to be associated with South Asian Ovalocytosis (SAO) (Nassar et. al, 1998) while reduction in abundance of band 3 anion transport protein was reported to be associated with Hereditary Stomatocytosis (HSto) ${ }^{(13)}$. CML is a progressive myeloproliferative condition marked by neutrophilia, and patients also suffer from moderate to mild anemia.

Fragmentation of band 3 bears a physiological significance as distinct fragments are senescent cell antigens, which elicit immune recognition and phagocytosis of aged erythrocytes $^{(14)}$. Band 3 profiles as a useful cumulative marker of oxidative and/or proteolytic stress conditions accelerated in stressful situations, such as inflammatory diseases, oxidative and proteolytic stress imposed by cardiovascular disease ${ }^{(15)}$. It has been reported that cathepsin G-mediated proteolysis of band 3 in CML erythrocytes (Govekar et al. 2012). Since distinct fragments of band 3 are recognized by serum $\operatorname{IgG}$ and thus induce erythrophagocytosis in vitro. This could exemplify a mechanism for the generation of eryptotic phenotype in $\mathrm{CML}^{7}$. Our results also could prove some evidence for it as there was no detectable band seen in the parallel area to $\mathrm{R}_{4}$ in CML patients as shown in figure I and an additional gel band was observed in $55 \mathrm{kDa}$ molecular weight area of CML patients (highlighted in arrow). Similar feature was observed in all the healthy controls and CML patients' samples used in this research. However, we were unable to detect or identify this band or the other fragment of Band $3(43 \mathrm{kDa})$ as stated in Govekar et al. (2012).

PMF and Mass Spectrometry associated technologies have facilitated fast and reliable detection and identification of proteins permitting mapping of protein profile of the mature erythrocyte. It has been shown that Govekar et al. (2012) used PMF and MALDI-TOF Mass Spectrometry to identify erythrocyte surface antigen bound by serum IgG. It proves that this MALDI-PMF technology still could be used in erythrocyte membrane research. Importance of this approach is well recognized by the fact that mRNA based microarray technology is not possible for erythrocyte proteome research. In this study it was possible to identify the above mentioned human ERMBPs that were separated by 1D-SDS-PAGE gel electrophoresis. Attempts to prepare a human erythrocyte membrane protein map using more advanced mass spectrometer techniques and complex protein identification tools are underway ${ }^{(16)}$. These new studies would undoubtedly result in identification of novel ERMBPs that will lead to comprehensive understanding of hematological diseases such as Polycythemia Vera, Leukemia, Sickle cell disorders, Thalassemia and Malaria ${ }^{(4,17)}$.

$\mathrm{R}_{\mathrm{X}}$ from healthy controls identified as identified as Dbf4 related factor 1 isoform 2 with a molecular weight of $18.60 \mathrm{kDa}$ and it was not detectable in CML patients. The annotation studies from Scaffold software and protein databases (www.uniprot.org) revealed that the main sub cellular location of this protein is nucleus and, it also present in intracellular membrane-bounded organelles. Dbf 4 related factor 1 isoform 2 has protein kinase activator activity and is believed to be activator of $\mathrm{S}$ - phase Kinase like proteins ${ }^{(18)}$. Binding of cell cycle regulated sub unit of Dbf 4 to Cdc 7 (Cyclin dependent kinase) is necessary for its kinase activity. Recent studies have shown conservation of Cdc7 - Dbf 4 kinases throughout evolution and their essential roles in DNA replication ${ }^{(19)}$.

Theoretically, mature erythrocyte does not contain any nuclear or organelle proteins unless it contaminated with white cells, platelets or serum. However, all the research groups those who succeeded in identifying large number of proteins have identified nuclear, endoplasmic reticulum, organelles or extra cellular proteins along with erythrocyte membrane/cytoskeleton proteins. Some of those were compatible with their molecular weight and others were higher or lower than the expected molecular weight ${ }^{(4,11,16,17)}$. Then the 
question that arises is "Are these proteins present in their active form?" Pasini et al. (2006) detected intracellular transport proteins of the Golgi network (eg, Rab 14, vesicle trafficking protein SEC22b, TPM 1, and syntaxin 7), the endoplasmic reticulum (protein disulfide isomerase A3), and the mitochondrion (heat shock protein $90 \mathrm{kDa}$ ).

Although RBCs are devoid of nuclei, a protein annotated to have transcription regulator activity, translation regulator activity, and chaperone regulatory activity was identified. Some of these proteins (elongation factor $1 \alpha 2$, eukaryotic translation initiation factor $2 \mathrm{C} 2$ ) are most probably in an inactive state as they are no longer needed in the absence of the nucleus and organelles and may be poly ubiquitinylated, which could be the reason of the increased observed molecular weight. Many of these proteins indeed had lower molecular weights than expected (ATP binding cassette subfamily B member 6, mitochondrial precursor) suggesting that they represented degradation products ${ }^{(11)}$.

Those results indicate that the degradation process initiated in reticulocytes is continued in erythrocytes, and it is likely that a lot of proteins identified in this study are still present in the mature cells while being in the process of being degraded. Therefore, more focused studies would be needed to test this hypothesis and verify whether some of the identified proteins are present in the $\mathrm{RBC}$ in an active state or represent leftovers from the degradation process and to elucidate in more detail the mechanisms of this process ${ }^{(11,17)}$. By analyzing Mouse and Human RBC proteome with the same technology as well as same methodology of sample preparation used in Pasini et al. $(2006)^{(11)}$, later the same group was able to confirm the unexpected RBC complexity ${ }^{(20)}$. In this study, the same organelle proteins occur in human and mouse RBCs. Apart from rare exceptions these are in the same metabolic state, suggesting that some organelle proteins may have (short lived) relevance even beyond the reticulocyte stage and share a common plan/schedule for metabolic degradation ${ }^{(16)}$. Their findings underpin the hypothesis ${ }^{(11)}$ that maturation of RBCs from reticulocytes and aging of RBCs are ongoing processes that continue their whole life span.

Table 1. Erythrocyte membrane associated proteins identified using following1-D SDS-PAGE separation and analysis by MALDI-TOF \& PMF profiles of normal healthy controls and chronic myeloid leukemia (CML) patients. $\mathrm{R}_{1}-\mathrm{R}_{10}$ protein bands (as shown in Fig. 1) present in both healthy controls and CML patients were analyzed. $\mathrm{R}_{\mathrm{x}}$ protein band is present only in healthy controls.

Number of peptides matching and \% sequence coverage is given for proteins identified using MALDI-TOF \& PMF. The identification spreads the broad molecular weight range

(from $31-281 \mathrm{kDa}$ ).

\begin{tabular}{|c|c|c|c|c|}
\hline $\begin{array}{c}\text { Gel } \\
\text { Band }\end{array}$ & $\begin{array}{c}\text { Protein identified } \\
\text { (Top Ranking) }\end{array}$ & $\begin{array}{c}\text { Accession } \\
\text { Number }\end{array}$ & $\begin{array}{c}\text { Sequence } \\
\text { Coverage (\%) }\end{array}$ & $\begin{array}{c}\text { Molecular } \\
\text { Mass (Da) }\end{array}$ \\
\hline R1 & Spectrin alpha chain, erythrocyte & P02549 & 15 & 281,043 \\
\hline R2 & Spectrin beta chain, erythrocyte & P11277 & 08 & 247,161 \\
\hline R3 & Ankyrin-1 & P16157 & 33 & 207,334 \\
\hline R4 & Band 3 anion transport protein & P02730 & 28 & 102,013 \\
\hline R5 & Erythrocyte membrane protein band 4.1 & P11171 & 20 & 97,528 \\
\hline R6 & Erythrocyte membrane protein band 4.2 & P16452 & 23 & 77,816 \\
\hline
\end{tabular}




\begin{tabular}{|c|c|c|c|c|}
\hline R7 & 55 KDa erythrocyte membrane protein & Q00013 & 25 & 52,492 \\
\hline R8 & Dematin & Q09485 & 34 & 45,600 \\
\hline R9 & Actin, cytoplasmic 1 & P60709 & 40 & 42,052 \\
\hline R10 & $\begin{array}{c}\text { Erythrocyte band 7 integral membrane } \\
\text { protein }\end{array}$ & P27105 & 32 & 31,882 \\
\hline $\mathrm{R}_{\mathrm{X}}$ & $\begin{array}{c}\text { Dbf4-related factor 1 isoform 2; } \\
\text { activator of S-phase kinase-like protein }\end{array}$ & Q8NFT6 & 36 & 18.600 \\
\hline
\end{tabular}

Table 2. Sequence information of the two matched peptides of identified protein spectrin, alpha chain, erythrocyte (Erythroid alpha-spectrin) gi/1174412/sp/P02549. The corresponding masses $\left[\mathrm{M}^{+} \mathrm{H}\right]$ for mono isotopic masses [M] of 1340.634 Da, 1545.701 Da in matched peptides are observed in the MALDI-TOF MS spectrum (Figure IIa).

\begin{tabular}{|c|c|c|c|c|c|}
\hline $\begin{array}{c}\text { Masured } \\
\text { Mass (M) }\end{array}$ & Ave./Mono. & $\begin{array}{c}\text { Computated } \\
\text { Mass }\end{array}$ & $\begin{array}{c}\text { Error } \\
\text { (Da) }\end{array}$ & $\begin{array}{c}\text { Missed } \\
\text { cut }\end{array}$ & Peptide squence \\
\hline 1340.634 & $\mathrm{M}$ & 1340.649 & -0.015 & 0 & LMEADIAIQGDK \\
\hline 1545.701 & $\mathrm{M}$ & 1545.727 & -0.026 & 0 & KHEAIETDTAAYEER \\
\hline
\end{tabular}

Table 3. Sequence information of the two matched peptides of identified protein spectrin, beta chain, erythrocyte (Beta-1 spectrin) gi/134798/sp/P11277. The corresponding masses $\left[\mathrm{M}^{+} \mathrm{H}\right]$ for mono isotopic masses [M] of 848.416 $\mathrm{Da}, 1113.520 \mathrm{Da}$ in matched peptides are observed in the MALDITOF spectrum MS (Figure IIb).

\begin{tabular}{|c|c|c|c|c|c|}
\hline $\begin{array}{c}\text { Masured } \\
\text { Mass (M) }\end{array}$ & Ave./Mono. & $\begin{array}{c}\text { Computated } \\
\text { Mass }\end{array}$ & $\begin{array}{c}\text { Error } \\
\text { (Da) }\end{array}$ & $\begin{array}{c}\text { Missed } \\
\text { cut }\end{array}$ & Peptide squence \\
\hline 848.416 & $\mathrm{M}$ & 848.431 & -0.015 & 0 & LMSELEK \\
\hline 1113.520 & $\mathrm{M}$ & 1113.538 & -0.018 & 0 & SYITKEDMK \\
\hline
\end{tabular}

\section{CONCLUSIONS}

In conclusion, we have shown that the PMF and 1 D-SDS-PAGE combined with MALDI-TOF-MS could be used to identify proteins as well as to determine the protein changes among CML patients and healthy persons. Recent technological advances have led to an in-depth and unambiguous characterization of erythrocyte proteins which include membrane bound and soluble cytosolic proteins. The technology used in this study is of relatively low accuracy and low resolution when compared to the recently developed technology. However authors believe that with the available resources at our disposal in this technique still holds promise to shedding light on ERMBP differences between healthy individuals and patients afflicted with hematological diseases related to erythrocyte 
membranes. It is also hoped that this initial research would at some point lead to discovery of biomarkers to these hematological diseases in Sri Lanka.

\section{Acknowledgements}

Financial assistance provided by International Science Programs (ISP), Uppsala University and technical support given by Ludwig Institute for Cancer Research, Bio Medical Center (BMC), Uppsala University, Sweden and Turku Centre for Biotechnology (TCBT), University of Turku and Åbo Academy University, Turku, Finland are kindly acknowledged. Facilities provided by Department of Physics and Plant Sciences, Faculty of Science, University of Colombo are also acknowledged.

\section{References}

[1] M. Almén, K. J. Nordström, R. Fredriksson, H. B. Schiöth, BMC Biology 7 (2009) 50.

[2] M. E. Reid, N. Mohandas, Seminars in Hematology 41 (2004) 93-117.

[3] A. V. Hoffbrand, J. E. Pettit, P.A.H. Moss, Essential Haematology (Fourth Edition) (2004) Wiley-blackwell.

[4] E. M. Pasini, H.U. Lutz, M. Mann, A. W. Thomas Journal of proteomics 73 (2010b) 421-435.

[5] M. Kundu, J. Basu, M. Fujimagari, P. Williamson, R. A. Chlegel, P. Chakrabarti, BiochimBiophys Acta 1096(3) (1991) 205-8.

[6] J. Basu, M. Kundu, M. M. Rakshit, P. Chakrabarti, BiochimBiophysActa (1988) 121-126.

[7] R. Govekar, P. Kawle, R. Thomas, S. Advani, P. V. Sheena, S. Zingde, Anemia (2012) 1-7.

[8] H. Lahm, H. Langen, Electrophoresis 21 (2000) 2105-2114.

[9] O. Vorm, P. Roepstorff, M. Mann, Anal. Chem. 66 (1994) 3281-3287.

[10] S. J. Cottrell, Journal of Proteomics 74 (2011) 1842-1851.

[11] E. M. Pasini, M. Kirkegaard, P. Mortensen, H. U. Lutz, A. W. Thomas, M. Mann, Blood 108 (2006) 791-801.

[12] T.Y. Low, T.K. Seow, C.M. Chung, Proteomics 2 (2002) 1229-1239.

[13] S. Shattil, B. Furie, H. Cohen, L. Silberstein, Haematology: Basic Principals and Practice. Edinburgh, Churchill Livingstone, (1995) 672.

[14] M. M. B. Kay, S. R. Goodman, K. Sorensen, Proceedings of the National Academy of Sciences of the United States of America 80(6) (1983) 1631-1635.

[15] A. Santos-Silva, E. M. B. Castro, N. A. Teixeira, F. C. Guerra, A. Quintanilha, Clinica Chimica Acta 275(2) (1998) 185-196.

[16] E. M. Pasini, H. U. Lutz, M. Mann, A. W. Thomas, Journal of proteomics 73 (2010a) 403-420. 
[17] F. Roux-Dalvai, A. Gonzalez de Peredo, C. Simo, L. Guerrier, D. Bouyssie, A. Zanella, A. Citterio, O. Burlet-Schiltz, E. Boschetti, P. G. Righetti, B. Monsarrat, Molecular \& Cellular Proteomics 7(11) (2008) 2254-2269.

[18] N. Yoshizawa-Sugata, A. Ishii, C. Taniyama, E. Matsui, K. Arai, H. Masai, The Journal of Biological Chemistry 280(13) (2005) 13062-13070.

[19] A. Montagnoli, R. Bosotti, F. Villa, M. Rialland, D. Brotherton, C. Merurio, J. Berthelsen, Santocanale, C. The EMBO Journal 21(12) (2002) 3171-3181.

[20] E. M. Pasini, M. Kirkegaard, D. Salerno, P. Mortensen, M. Mann, A. W. Thomas, Molecular \& Cellular Proteomics 7 (2008) 1317-1330. 